Meta

Journal des traducteurs

Translators' Journal

\title{
... Les opérations transformationnelles de Louis Wolfson
}

\section{Françoise Quaré}

Volume 27, numéro 1, mars 1982

Psychanalyse et traduction

URI : https://id.erudit.org/iderudit/003882ar

DOI : https://doi.org/10.7202/003882ar

Aller au sommaire du numéro

Éditeur(s)

Les Presses de l'Université de Montréal

ISSN

0026-0452 (imprimé)

1492-1421 (numérique)

Découvrir la revue

Citer cet article

Quaré, F. (1982). ... Les opérations transformationnelles de Louis Wolfson.

Meta, 27(1), 93-98. https://doi.org/10.7202/003882ar d'utilisation que vous pouvez consulter en ligne.

https://apropos.erudit.org/fr/usagers/politique-dutilisation/ 


\section{...LES OPÉRATIONS \\ TRANSFORMATIONNELLES \\ DE LOUIS WOLFSON}

FrançoIse QuARÉ

Parler de ce livre «Louis Wolfson: Le schizo et les langues", à des linguistes c'est avant tout répondre au vœu de l'auteur.

Il n'aime pas du tout les psychanalystes et les tourne en dérision pour leur connivence à son endroit avec la psychiatrie. Qu'il s'agisse de nosographie, d'interprétation papa-maman, et de neutralité bornée, son style vaut comme parodie cinglante de leurs études de cas et leur démontre qu'il pourra toujours leur en remontrer sur sa propre présentation de malade.

Quant aux linguistes, il les aime et s'autorise de la passion qu'il partage avec eux pour leur adresser l'interprétation qu'il se découvre à la fin du livre :

Aussi, le schizo s'imagine de fois à autre avoir découvert un facteur émotif, sans doute plus ou moins subconscient, car il ne le trouvait jamais mentionné dans un livre, ! mais, il n'a certainement pas beaucoup lu, lequel facteur, entre autres, pousserait les gens à étudier la linguistique générale et en particulier la grammaire comparée, et ça serait d'après lui un désir, peut-être vague, sinon subconscient et refoulé, de ne pas devoir sentir leur langue naturelle comme une entité, comme la sentent les autres, mais par contre de pouvoir la sentir bien différemment, comme quelque chose de plus, comme exotique, comme un mélange, un pot pourri de divers idiomes.

Il leur rend à eux aussi ce service de subvertir le sujet de la linguistique comme science, mais surtout d'opérer cette subversion à partir d'une pratique tout ce qu'il y a de plus originale de la linguistique. Ça serait trop facile de s'en distraire comme d'une fantaisie ou d'un spécimen clinique.

Ce que je veux vous transmettre du point où ce texte rencontre mon expérience - même s'il ne s'agit que de ma lecture de ce texte - c'est que l'original de cette pratique de linguiste est de faire acte d'interprétation pour lui comme pour eux, comme pour nous, et d'élaborer cet acte tout au long de ce livre.

Cette linguistique est donc à prendre au mot, je veux dire, aux mots qu'elle retient comme exemples et à leur ordre de succession dans le temps, je veux dire de leur découverte au fil du texte.

Je m'autorise finalement à suivre la progression des chapitres comme une suite de découverte de mots. Plus précisément comme une production de signifiants faisant chaîne après coup. 
Alors qu'à une première lecture j'appréhendais le livre dans son entier comme une arme linguistique, aux dires même de l'auteur, contre la langue qu'il trouvait souvent tellement agaçante, à savoir l'anglais, je parcourais rapidement les paragraphes plus techniques de transformation de mots, comme on lit des exemples d'une grammaire, tous plus ou moins sur le même plan, comme des illustrations choisies pour leur capacité à faire valoir telle ou telle loi morphologique, dans un temps de démonstration grammaticale, temps apparemment sans histoire.

Et puis voilà qu'au moment d'en écrire quelque chose moi-même pour vous le dire j'ai vu se creuser un écart entre cet état de guerre toujours recommencée dans une durée immobile, et son travail d'écriture proprement linguistique. Autrement dit, à un premier niveau, celui du récit, son ennemi est la langue anglaise et à un second niveau, certains mots seulement sont à la lettre inaudibles et son travail linguistique est de les détruire. Le premier niveau est celui de la création d'un imaginaire, tandis que le second permet une symbolisation. Alors que le récit semblait porter la chronologie autobiographique, je découvrais que le temps ne s'accroche véritablement qu'au rythme des mots qui s'ordonnent sous couvert d'exemples et tombent successivement pour faire chaîne. C'est donc au moment précis pour moi d'écrire ceci que ces mots-exemples ont pris tout à coup leur relief d'écriture efficace, mots magiques, incantatoires aux dires mêmes de l'auteur, embrayant un autre temps que celui des morts-vivants. Mots interprétations dirons-nous, chacun comme tel valant rétroactivement par rapport au suivant. Ils perdent alors radicalement leur statut d'exemple pour prendre le pas sur le récit qui semblait justifier leur apparition. Le récit dès lors devient ce qui rend accessible au lecteur le passage de l'inaudible à l'inouï.

Je vais à mon tour m'exercer à ce passage en amorçant la séquence de ces mots.

1. Nous avons dit qu'à un premier niveau les langues permettaient la création d'un imaginaire - ce qui semble paradoxal pour un outil du symbolique.

Le premier mot énoncé est TREE - qui a une fonction inaugurale, celle d'engager le schizo, comme il se nomme, dans sa recherche linguistique, c'est-à-dire un autre rapport aux langues que celui de leur simple étude pour les bien parler ou les bien traduire. Il est énoncé par son père en tant que ce mot serait pour lui le même en russe et en anglais.

Disons tout de suite qu'il s'agit là tout d'abord de faire exister un dehors plus ou moins allié par rapport à un dedans ennemi là où c'était indifférencié omnipotent la langue anglaise soi-disant universelle et donc illimitée. Ce sera le premier mot charnière, ou d'aiguillage, ou de passe, comme on voudra, celui qui dira: là une limite peut passer. Limite pas seulement pour lui, mais aussi pour l'autre incarné par son père.

Ce mot apparaît au chapitre 2, après que nous avons fait le tour de tous les schémas explicatifs qu'il nous suggère à sa compulsion irrépressible à l'étude des langues. Explication psychologique : c'est une réaction à une difficulté scolaire - il ne pouvait ni épeler ni lire. Explication psychiatrique : il a des antécédents chargés, beaucoup de fous dans sa famille. Explication sociolo- 
gique : les migrations des juifs d'Europe Centrale leur ont fait abandonner à la frontière leur langue véritablement maternelle, pour l'anglais, celle du colonisateur. Tous ces schémas sont de causalité. Mais c'est à une autre discipline qu'il va nous entraîner.

Ce mot lui fait découvrir qu'il existe un passage possible d'une langue à l'autre, un lieu interlangue où peut passer la guerre et aussi l'échange. C'est aussi la preuve, le témoin que pour son père un ailleurs de l'anglais existe, et c'est cela qui le constitue comme autre. En effet, on s'aperçoit que s'il ne parle qu'allemand à son père, ça n'est pas seulement pour fuir l'anglais mais surtout pour le faire parler yiddish - c'est-à-dire aussi bien pour le faire parler autre chose, russe par exemple, que son père se défend de parler : «ça ne sert à rien, seul l'anglais est utile». Peut-être veut-il faire que son père se souvienne de ses racines perdues, dans un retour vers l'orient. Mais il s'agit surtout de le forcer à sortir de sa léthargie de clochard et à construire pour son fils un dehors et un dedans par rapport à ce qui était jusque-là la langue indifférenciée de tous les autres.

Comment se passe cette séance de ce jour-là : il y va de tous ses symptômes (grimaces; il se bouche les oreilles, il regarde ailleurs), pour faire sauter la résistance où son père se réfugie : «le russe ne me servirait à rien. En anglais on est compris de partout ». Il suscite ainsi un état de tension telle que, exaspéré, le père finit par lâcher: «maintenant tu peux retirer un moment tes doigts de tes oreilles... J'ai quelque chose de très important à te dire... J'ai appris quelques mots d'anglais avant que je sois venu ici de la Lituanie... Tu peux même apprendre le russe... vois tous les arbres là-bas! et bien en russe on dit arbre tout comme en anglais TREE.»

Plus tard il découvrira que cette similitude était fausse, mais peu importe; le mot aura fait son effet de mot frontière faisant exister un extérieur de la langue, effet de mot contigu, frontalier, qui de l'anglais même crée un ailleurs accessible. Ailleurs jusque-là non pas inaccessible mais inexistant. Ce n'est qu'après coup, après l'avoir fait exister qu'on le dit inaccessible ou dissocié.

Le dissocié cher aux analystes est un artefact, et non quelque chose qui était là avant, inaccessible comme le refoulé ; il est un moment de remaniement de l'imaginaire qui laisse la possibilité d'accès à de nouveaux systèmes d'opposition jusque-là impensables, et comment? par un forçage de l'autre bien obligé d'opposer de nouvelles balises imaginaires à l'irruption du réel que constitue tout rapport psychotique. Ainsi, le dissocié ne fait son apparition qu'au moment même de la possibilité de constituer deux bords et de les délimiter. Comment? Par un signifiant que nous avons appelé de déprise de ce rapport. On peut le penser dès lors comme existant ailleurs, accessible à la conscience mais clivé. Nous appelons ce clivé le rêve de l'autre, l'autre construit provisoirement pour donner cadre pour un autre à ce qui est autrement irreprésentable, sans aucun autre pour en répondre, à savoir le réel. C'est alors qu'il révèle son étrangeté comme inquiétante; jusque-là tout était normal, la folie même, il n'y avait plus qu'à en mourir.

2. Le second mot est SHORTENING. Il apparaît au chapitre 3. C'est le chapitre où entrent en scène sa folie et son moyen d'en sortir. 
Tout va très vite. Comme une brusque pulvérisation des bagages du corps. L'ail est envahi par une vision informe - sa mère fait irruption - l'oreille est assourdie par un fracas innommable - et s'ensuit une orgie de nourriture bourrant et contusionnant sa bouche devenue trou, et lui machine à manger automatiquement pour une durée limitée mais inconnue.

Il faut lire la description de ce qui se passe à partir de cette entrée en folie pour se trouver plongé dans une dimension non identifiable où se passe quelque chose qui n'a pas de nom, plaisir affreux. Parler là de symbiose, de jouissance, c'est mettre justement des mots trop commodes à nous rassurer dans nos propres repères - inévitables par ailleurs - comme si on savait de quoi il s'agit quand il n'y a plus rien pour faire exister le dedans par rapport au dehors. La chose par laquelle il est happé, c'est à lui de la qualifier.

C'est en la qualifiant qu'il s'en déprend par son travail linguistique.

Techniquement comment s'y prend-il ?

Il va chercher sur les emballages de nourriture un couple de mots : VEGETABLE OIL, mot magique, mot de passe comme TREE dans deux autres langues, le français et l'allemand, pour son homophonie dans les trois langues. Mot inoffensif, et même plus enchanteur car il libère une chaîne associative. Il permet d'accéder par métonymie à VEGETABLE SHORTENING.

De façon mystérieuse mais très explicite, shortening est défini comme le premier mot à anéantir en en faisant l'analyse quasi chimique. Avant tout, il attire sur lui comme la foudre tout le monstrueux de la situation évoquée plus haut, comme si en l'espace concret ultraréduit de tous les mouvements phonatoires qui en permettent la prononciation, allait se jouer sa transmutation en limite.

D'abord par double sens, le sens de "graisse à pâtisserie" s'efface au profit du sens «abréviation». Mais aussi par analyse phonétique de l'adjectif SHORT «résidu» qu'il fait sonner dans toutes les langues exotiques à sa disposition, en attirant rétroactivement sur lui les connotations sémantiques de ces autres langues. Il restitue ainsi au «SHORT», d'abord pur bruit (nous disions réduction de l'espace de la déflagration), des sens prédicatifs qui endiguent la chose à l'œuvre dans cette folie.

Alors, nous pouvons commenter, mais ça n'est déjà plus très important : - brusque est le prédicat des irruptions : «à pas de loup elle entre en éclair et tonnerre» - court est celui des ingurgitations atroces «en avaler le plus dans le plus court délai» - maigre de l'allemand schmall, du français chétif est le premier adjectif du livre qu'il ouvre de son corps : "le jeune homme schizophrénique était maigre comme beaucoup de gens dans de tels états mentaux».

Où la maigreur du corps s'érige dès lors en limite de ce que nous appelons pour nous l'autre corps réel du rapport psychotique. Le gras et le maigre, pour parodier Lévi-Strauss, introduit un autre type d'opposition que celle qui joue entre les langues. Il s'agira dans un autre registre d'opérer sur certains mots un processus de symbolisation.

3. Le 3 e mot est LADIES, au chapitre 4, celui de la musique. Après qu'a été comme jugulé le moment de folie par la production de l'adjectif SHORT, 
il y a un intérieur possible à de tels moments et on peut à la lettre l'écouter comme quelque chose d'à vif, une plaie hurlante dont son corps est le bord extrême, la membrane extérieure de cette chose brusque et bruyante. Il écrit ce livre au moment où il cesse justement d'être pure surface d'impact de ces bruits pour les transformer en signifiants.

Le mot LADIES est le second à subir cette transformation. Hurlé par sa Lady de mère dans une chanson, (Good night ladies), il est à la lettre inaudible, mot-chose jeté dans les tympans, mot-bruit non situé au lieu de l'Autre comme signifiant. Pour qu'il accède à ce statut il va falloir le décrire comme bruit. Le nom russe choisi LOUDI est retenu plutôt que l'allemand LEUTE pour la musique de sa déclinaison qui le restitue dans sa dimension de voix et permet un travail de tonalité, pas seulement de souffle articulé comme dans shortening.

Le privilège ici est donné à l'Accent, à la musique de la voix qui fait parler l'au-delà. L'au-delà où, à ce moment-là, l'opposition, soi-disant reçue de tous, entre LADIES et MEN est tout simplement escamotée. Remaniement de l'imaginaire, disions-nous, qui va aussi bien dans le sens de créer de nouvelles oppositions que d'en gommer d'autres par rapport auxquelles il n'a pas, pour ce moment-là, à se repérer. Par contre la musique et la voix sont repères de corps.

4. Le chapitre de la musique est donc, dans cette logique, suivi du chapitre de l'oubli.

Le $4^{e}$ mot retenu est WHERE: «la mère du schizophrène fréquemment oubliait où elle avait temporairement placé quelque chose... Où sont mes lunettes? hurlerait-elle soudain par exemple... Quoiqu'il en fût, l'étudiant de langue schizophrène trouvait bien incommodes ses tentatives compulsives et obsédantes de tourner de manière instantanée et définitive WHERE en allemand WO le mot anglais lui semblant au contraire toujours sauter et rebondir avec opiniâtreté dans sa tête.»

Cette gêne cessera lors de l'illumination qui le fera tout simplement remplacer WHERE : OÙ, par l'allemand WOHER : d'où... (il éprouva une sensation même mêlée de joie - de vrai accomplissement... qu'il était satisfait de lui-même, qu'il avait des idées.»

Mais gardons-nous d'emboîter le pas à son euphorie pour plaquer nos catégories sur cette sensation, et de chercher à y reconnaître celle qui dans la doxa analytique accompagne la levée de barrières, celles du refoulement par exemple, lors de l'advention du JE-là-où-c'était. Le chapitre suivant nous conduit à préciser ce qu'accompagne cette sensation.

5. Le chapitre 6, du téléphone, fait apparaître les mots: I KNOW-YES et CALL.

Il y est question du savoir, du lieu de l'appel et de la supposition. Et c'est par ce biais des appels téléphoniques que des mots familiers de la théorie analytique sont promus au travail linguistique.

- I KNOW - le verbe du savoir, dont il ne dit pas qu'il y a un savoir insu du sujet, mais que, dans un certain sens, personne ne sait rien, et peut-être en particulier sa mère; il est remplacé par le verbe russe ZNAIOU je sais. 
- YES - jouant sur le double sens du français si, est balayé comme affirmation par le si conditionnel : rien ne peut être affirmé, tout ne peut être que supposé.

Or ce qui est supposé par son travail et justement par cette chaîne de mots, c'est qu'il y a quelque part de la forclusion, de quoi? on n'en sait rien, puisque là nul savoir n'existe: «I know», même pas supposé puisque là aucune affirmation «yes», aucune Bejahung primordiale n'a pu se faire.

Et cet impressionnant dispositif de travail sur les langues est là comme repérant à tâtons la possibilité de cette affirmation, là où la forclusion est supposée, là où l'autre chancelle, voire se dissout.

L'illumination et la satisfaction de ses trouvailles que nous disions comparables à des levées de barrières, signalent plutôt le surgissement de telles barrières. Elles permettront, dans une mise en scène opposant la langue haïe et les langues bannies, que se joue tout simplement un meurtre. Le meurtre de motschoses comme dans un refoulement originel : URVERDRÄNGUNG qui permet la naissance de signifiant. Le cri qui envahissait l'espace sonore devient alors voix d'appel et de reconnaissance. KERIA.

Le dernier mot de ce chapitre à être assassiné est CALL, devenu l'hébreu

Il faudrait continuer à suivre le déroulement de cette alchimie du verbe où Louis Wolfson s'autorise d'une position analytique pour inventer ce qu'il faut bien appeler une subversion de la folie. $\AA$ quoi sa linguistique donne forme et corps de théorie. 